\title{
Comparison of router testbeds: embedded system-based, software-based, and multiprotocol label switching (MPLS)
}

\author{
M. H. M. Nasir, N. A. M. Radzi, W. S. H. M. W. Ahmad, F. Abdullah, M. Z. Jamaludin \\ Institute of Informatics and Computing in Energy (IICE), Electronic Communication (EC) Department, \\ College of Engineering (COE), Univerisiti Tenaga Nasional, Malaysia
}

\begin{tabular}{|c|c|}
\hline Article Info & ABSTRACT \\
\hline Article history: & Multiprotocol Label Switching (MPLS) is a promising packet switching \\
\hline Received Dec 1, 2018 & $\begin{array}{l}\text { technology in the field of communication network, allowing router to } \\
\text { forward the packets based on labels. However, off-the-shelves routers are }\end{array}$ \\
\hline Revised Mar 3, 2019 & made to be non-configurable, making it less suitable for academic research \\
\hline Accepted Apr 24, 2019 & $\begin{array}{l}\text { purposes. The aim of this paper is to explore the possibility of developing a } \\
\text { configurable MPLS router by reviewing embedded system-based router, }\end{array}$ \\
\hline Keywords: & $\begin{array}{l}\text { software-based router and commercial MPLS router itself. The architecture } \\
\text { of the routers will be described in detail and their performances will be }\end{array}$ \\
\hline $\begin{array}{l}\text { Embedded system-based tesbed } \\
\text { MPLS router } \\
\text { Router testhed }\end{array}$ & $\begin{array}{l}\text { compared. From the analysis, we found that the development of } \\
\text { reconfigurable MPLS router testbed is achievable by using embedded } \\
\text { system-based hardware due to its freedom of reconfigurability. }\end{array}$ \\
\hline
\end{tabular}

Copyright @ 2019 Institute of Advanced Engineering and Science. All rights reserved.

\section{Corresponding Author:}

Muhammad Haqeem bin Mohd Nasir,

Institute of Informatics and Computing in Energy (IICE),

Universiti Tenaga Nasional,

Jalan Ikram-Uniten, 43000 Kajang, Selangor Darul Ehsan, Malaysia.

Email: M.Haqeem@uniten.edu.my

\section{INTRODUCTION}

MPLS is an evolving connection-oriented switching technology based on labels instead of Internet Protocol (IP) address and is an undergoing study until today [1-7]. This technology creates virtual connections between two Label Edge Routers (LER) that are located at the edge of backbone network [8]. The backbone of the MPLS network consists of LER, Label Switch Router (LSR), and Label Switch Path (LSP). One role of LER is for Quality of Service (QoS) to ensure and distribute the labels to all the network components along unidirectional tunnel called the LSP [8-9]. It is also the first router to encapsulate and decapsulate by adding and removing labels onto the packets into the LSP and make the initial path selection.

According to Shawl et al., the LSR comprises Label Distribution Protocol (LDP) that will be applied to the LSP as the signaling protocol for distributing labels throughout the MPLS network [9]. LSR has two main functions. Firstly, it is to ensure that the LSP is created. Secondly, it ensures that the data is transmitted according to the appropriate routing tables of labels that are created by LSR itself. When the LSR received a packet, it checks the routing table to find the next hop which is also an LSR. Then, it detaches the label and attaches a new one before it sends packet to the next hop $[8,10]$.

Currently, MPLS technology is meant for industrial purposes such as in teleproctection, Supervisory Control and Data Acquisition (SCADA), and protection relays to achieve low latency and high reliability [11-15]. Hence, the label switching mechanism can be configured freely only by the manufacturers and vendors. At the user side, MPLS routers can be configured based on limited options provided by the vendor to make sure the router is not vulnerable to hacking.

Therefore, the freedom of reconfiguring of MPLS router is necessary for academic research purposes. Due to that reason, various of studies have been done by using simulation, commercial MPLS 
router and embedded systems. This paper is aimed to compare and analyse the existing commercial MPLS routers and embedded system routers in terms of hardware architectures and performances which will be useful for developing the reconfigurable router testbed and benchmarking its performance.

The remainder of this paper is structured as follows; Section 2, we discuss on the related work of previous router testbed. It is divided into three parts which are software-based, commercial MPLS router, and embedded system-based router. Then, in Section 3 and 4, the performance of commercial and softwarebased MPLS router and embedded system-based routers are analyzed respectively. Finally, this paper is concluded with Section 5.

\section{RELATED WORK}

Various studies on reconfigurable routers using software simulations, commercial routers and embedded system-based routers are reviewed in this section. The routers will be described in detail in terms of architecture, structure, and cost effectiveness. The summary of these work will be tabulated at the end of this section.

\subsection{Software-based Router Testbed}

This section presents studies that have been conducted to develop a software-based router testbed. The main simulators in the literature are Click [16-17], Minos and Data Plane Development Kit (DPDK) [18], MATLAB [19] and C++ [20].

In 2013, Aldaya et al. [16] created a programmable optical router to test optical switching fabrics by using SwitchingMatrix in Click software. Wang et al. [17] further improved Click-based router testbed by proposing an additional software which is OpenFlow to their testbed. The testbed achieves high speed packets switch forwarding as it utilises personal computers and hubs. Hence, a load-balancing highperformance router testbed can be achieved and it can be expanded to support network virtualization. This software-based router testbed is also modular, making it easy to conduct network experiment in the laboratory for academic purposes.

In a different work by Xu et al. [18], a framework called Minos has been proposed to regulate router actions on data planes. Action Identifier (AID) will be the input for Minos to perform lookups in Regulated Action Table (RAT). Finally, Minos will verify whether or not the action is normal. Minos is expected to get a couple of distinct factors that will affect the security of a programmable router such as cost and effectiveness. This is because the reprogrammability makes the router vulnerable and exposed to the risk of being hacked. Minos is implemented and evaluated separately on Click and DPDK. DPDK comprises of a set of libraries that support efficient implementations of network functions [21].

The worked by Tajiki et al. [19], focuses on a new traffic engineering architecture for SDN-MPLS network, where they proposed to improve flow-level management flexibility. It is done by applying OpenFlow-enabled switches at the edge of the network while the MPLS routers are the core router of the network. The proposed scheme also re-assigns flows in the LSP to highly utilize the network resource. The simulation was done by using MATLAB2016b.

The software router by Goswami et al. [20] studied how to achieve the estimation of optical-layer power consumption and cost for a long distance optical networks using Wavelength Division Multiplexing (WDM). The test is implemented on two different networks which are IP-over-WDM and IP-over-MPLSover-WDM networks. In the former network test which is IP-over-WDM network, IP routers communicate directly with Wavelength-Routed Optical Network (WRON) and bandwidth requests are equal to light-path capacity. On the other hand, in IP-over-MPLS-over-WDM network, the IP routers communicate with WRON through commercial routers, hence the bandwidth requests arrived as LSP. Therefore, it is lower than lightpath capacity. The simulation was conducted by using $\mathrm{C}++$ programming language. The advantage of this work is that it is cost efficient to run the simulation. However, the results obtained are based on theoretical values because there is no hardware involved.

Despite the fact that it is costs efficient to develop a software-based router, but the parameters in the simulations are based on theoretical values. Meaning that, the simulations does not consider noise, non-linear effects, and other environmental factors like hardware does. Therefore, hardware emulation is a usual progress of testbed development.

\subsection{Commercial Router}

A review of commercial routers will be described in this part. One of the previous work, Software Defined Network (SDN) is hybrid with commercial MPLS routers in [22]. The only work that used commercial routers alone is by Blair et al. [23]. 
In a work by Sgambelluri et al. [22], Segment Routing (SR) is implemented in two different networks, which are SDN-based and Path Computation Element (PCE)-based. SR technology is proposed to enforce an effective routing strategies without relying on signaling protocols. In SDN-based network, SR controller is designed to enhance OpenFlow controller. While in PCE-based network, SR is implemented where the nodes consist of commercial IP/MPLS routers. This work analysed the performance of both proposed implementations.

Blair et al. [23] used a commercial MPLS router in his study. The authors used four MPLS routers in their experiment, with multiple protocols such as IEEE C37.94, IEC 61850-9-2 Sampled Values, and IEC 61850-8-1 Generic Object-Oriented Substation Event (GOOSE). The study is to demonstrate and analyse the use of commercial IP/MPLS protocol to carry protection relay hardware to support power system protection functions. They also used Real-time Digital Simulator (RTDS) to simulate the power system and interfaced with the hardware protection relays. RTDS allows power system faults and other events to be simulated in real-time.

\subsection{Embedded-System based Router Testbed}

The design of embedded system-based router testbeds were mostly designed using Banana Pi [24], netFPGA [25] and Raspberry Pi [26]. The main purpose of using embedded system as a router is to create a reconfigurable router testbed unlike commercial router.

Posch et al. [24] proposed a testbed using Banana Pi R1 as a router. Banana Pi is a single-board computer that has more than one Ethernet ports. This work demonstrates a Named Data Networking (NDN)based multimedia delivery using adaptive bit-rate streaming. Additionally, a graphical user interface is provided for the visitors who watch the demonstration to create their own network topology, configure a streaming scenario and observe in near-real time. The visitors may also learn which configuration gives the best performance of NDN network by selecting different network forwarding strategies and client-based adaptation logics. As for the hardware, they used Banana Pi R1, at least two network switches, and a personal computer (PC). The testbed required a large space due to large number of components. Banana Pi R1 is using A20 ARM Cortex A7 Dual Core as its processor. Then, two dedicated networks which are Management Network (MN) and Emulation Network (EN) are connected with Banana Pi R1 in a star topology. The role of Management Network (MN) is for configuration and monitoring. While EN is a virtual network overlay that is created using networking tools such as iptables and traffic control. The purpose of having the separation between $\mathrm{MN}$ and $\mathrm{EN}$ is to prevent management and control interference with running network emulation. However, according to Lech and Włodarski [27], Banana Pi has its own weakness. It produces a huge delays (up to $43 \mathrm{~ms}$ ) for such a simple point-to-multipoint Local Area Network (LAN) and Wide Area Network (WAN) topology. Furthermore, according to the author, Banana Pi can cause a serious problem to the network when it receives a large amount of traffic.

Another study done by Sivarman et al. [25] used netFPGA as their router to study the role of packet buffer memory on the power consumption of backbone routers. They have developed an algorithm for the memory components to sleep and awake when needed, while being able to control the resulting traffic performance degradation in the form of packet loss during congestion. They conducted a comprehensive evaluation of their algorithm by using the simulation of offline traffic traces taken from carrier/enterprise networks as well as online TCP flow in Network Simulator 2 (NS2). The evaluation also being implemented on a programmable router testbed which is the netFPGA. NetFPGA is connected to traffic generators and delay emulators to demonstrate the feasibility of implementing the algorithm in the hardware. The advantage of using netFPGA is it can be reprogrammable by using VHDL programming language. However, the cost of netFPGA is high.

Gupta et al. [26] has proposed a small size, low cost and portable SDN switch testbed using Raspberry Pi. Raspberry Pi is a minicomputer utilizing Advanced Reduced Instrcution Set Computing Machine (ARM) as its CPU [28-29]. The testbed reported by Gupta et al. [26] claimed to support OpenFlow Specification 1.0 to 1.4. However, the Raspberry Pi only has 1 Ethernet port but the authors extend it using three low-cost USB-based LAN cards. In order to make the Raspberry Pi as SDN switch, it is installed with Ubuntu MATE 15.04 instead of Raspbian to support the latest version of OpenFlow switch. Then, four laptops are connected to the SDN switch as client, POX/RYU controller, the other two laptops as servers. The advantage of using Raspberry $\mathrm{Pi}$ as the testbed is it is easy to configure in terms of installing the operating system, programming language and the user interface. The price of the Raspberry $\mathrm{Pi}$ is also reasonable and suitable for beginners. However, the only downside of Raspberry Pi is it only has one Ethernet port, therefore it is quite hard to emulate a real network router. 


\section{PERFORMANCE OF COMMERCIAL ROUTER}

The results and performance of the hardware and software-based MPLS routers testbeds will be presented in this section. The parameters such as throughput, delay, link utilizations, bandwidth and power consumption will be discussed in detail.

In segment routing (SR) work by [22], SDN-based SR controller uses OFP_FLOW_MOD message to configure the nodes while PCE-based SR controller uses PCRep to configure the nodes which are the IP/MPLS routers. The scalability performance is tested in two different cases which are single label and 15 labels. The overall results show that the controller setup time, ingress switch setup time and packet forwarding time for 15 labels are greater than single label. This is due to a lot of time required for path and label stacking computation. As for PCE-based SR controller, the results are in terms of distribution of PCE service time. Comparing with SDN-based SR controller, the two results are similar because the same path and segment list computation were implemented in both controllers. However, for both implementation, SR controller does not provide the router testbed a rerouting mechanism because it is based on shortest path routing. Hence, if the shortest path is congested, the router will always forward the packets on that path.

Lastly, for work done by [23], the author runs two different experiments. The first experiment is to study the delay and jitter of IEEE C37.94 protocol. While the second experiment is to study the comparison of protocol trip times and bandwidth between IEEE C37.94 and IEC $61850 \mathrm{SV}$ and GOOSE. For the first experiment, the author runs the experiment for nine times. The first five tests are to get the propagation delay results for a chain of IP/MPLS routers. According to the results, the jitter buffer size increases as the payload size increases. Thus, the propagation delay increases. For sixth test onwards are to get the results of propagation delay for ring of IP/MPLS routers. The advantage for the ring setup in sixth test compared to fifth test is it has rerouting capability because the setup has an alternate path for the packets in case of broken link. There is small difference in terms of propagation delay for the sixth and seventh test due to the variation of delay in the hardware. At the eighth and ninth test, the propagation delay increases when number of C37.94 slots decreases even when the payload size is very small. This is because the main purpose of the slots is to define the end-to-end usable bandwidth for the packets. Hence, if the number of slots is small, the usable bandwidth will also be small causing the delay to be high. For the second experiment, the results show that IEC $61850 \mathrm{SV}$ and GOOSE are better than IEEE C37.94 in terms of circuit breaker tripped time, and backup intertrip time. Even the bandwidth used by these two protocols is greater, they are able to transfer much more information for three-phase voltage and current waveforms. Hence, the data acquired is more accurate and better for further analysis.

Therefore, from the performance of the MPLS routers above, we can conclude that MPLS routers are better than IP routers because it is scalable [22], protection stability [23], improves bandwidth efficiency [23], and low value of delays.

\section{PERFORMANCE OF EMBEDDED SYSTEM-BASED ROUTERS}

This section will present the findings obtained by the embedded system-based router from Section 2.3, and will be compared in terms of throughput, cost, and processing capability at the end of this section.

The study carried out by [24] have successfully developed a low-cost router testbed based on Banana Pi R1. The testbed can be accessed by Virtual Private Network (VPN), thus the configuration, execution and the visualization of the testbed did not require a lot of bandwidth. The multimedia delivery scenario can be configured by using the Web interface. In terms of delay, their results presented in [30] show that the delay is big despite the packets are sent from one node to its adjecent node via CAT6 Ethernet cable. This is because the Banana Pi R1 is connected with two networks, hence the processor cannot keep up with the networks, operating systems, and data transmission at the same time. Supported by the results obtained in [27], it shows that Banana Pi R1 gives low throughput and high delay for point-to-multipoint connection in LAN and WAN network despite it has Gigabit Ethernet ports. To overcome these limitations, suggested method to reduce the delay is to reduce the number of nodes because the higher the number of nodes, the greater the delay. Furthermore, Banana Pi R1 is suggested to connect to the network via fiber optics instead of wireless and Ethernet. This is because fiber optics provide more effective solution for long distance data transmission. In terms of topology, the nodes in [24] are connected in star topology. The advantage of using this topology is the client can have point-to-point communication with other client resulting the transmission delay is lesser than the other topologies. However, this type of topology does not have an alternate route for the packets in case of broken links. Hence, an improvement that can be made is to use ring topology to prevent data transmission failure in case of broken link as well as link congestion.

While in [25], the power against loss trade-off performance were evaluated when the algorithm is implemented on netFPGA and TCP flows were generated for 180 seconds using iperf. The algorithm has saved $40 \%$ of the energy when it is under very heavy load. As a comparison, for example, a Raspberry Pi 
typically can generate around $98 \mathrm{Mbps}$ for 10 seconds. Which means, it can generate a huge amount of throughput within short period of time compared to netFPGA. This is due to some packet loss as described in their work.

The most recent work in [26] did not present any numerical result. However, they have successfully developed and tested the SDN-switch by using load balancing and firewall application. The load balancing architecture consists of OpenFlow switch network with POX controller and multiple servers connected to the OpenFlow switch. The firewall allows or rejects a specific type of data. In this case, the firewall only allows MAC address, IP address, and port number. When the packet arrived at the switch, the firewall check whether the packet header matched with its rules or not. The main advantage of this testbed is the hardwares are cost friendly since Raspberry Pi were being utilised with a few personal laptops to run the experiment. However, this testbed is expected to have a high delay because only one Raspberry Pi is connected to all the laptops. This will cause a problem for the Raspberry Pi to keep up all with the data that are transmitted at the same time. Furthermore, the topology is also the same as [30] which is star topology.

From the performance of the said embedded system-based routers, each of the testbeds have their own pros and cons affected by the type of traffics, topology and hardware. From the analysis, Raspberry Pi is a better choice for a cost-friendly testbed [26], because it can generate high throughput within short period of time and it can handle multiple nodes connected to it.

\section{CONCLUSION}

This paper has presented an overview on router testbeds of software-based, commercial, and embedded system-based. These testbeds have similar method of conducting the experiment which are using hardwares, softwares, or both. For the software-based testbeds, the simulations achieved customizable, programmable, and has the capability to emulate a complex routing network while achieving a highperformance testbed. However, the results obtained are based on ideal case and not involving non-linear effects. As for commercial routers, the results show that it is better than traditional IP-based routers because it can provide better throughput, protection, bandwidth and link efficiency, and delays despite it consumed higher power. However, commercial routers do not have the freedom to reconfigure for academic purposes. Last but not least, design of a completely reconfigurable and reprogrammable router testbed can be achieved by using embedded system hardware making it the most suitable choice for academic purposes. However, they have limited processing unit capability causing the performance of the network is lesser than using industrial routers. Therefore, our next study is to develop a reconfigurable MPLS router testbed by using embedded system-based hardware, which will be utilising analysis from this paper as the basis. The MPLS router will be useful for architecture and performance benchmarking. Hence, the plan for the future work is to validate the performance results of the developed reconfigurable router with the industrial grade MPLSbased router.

\section{ACKNOWLEDGEMENTS}

The author gratefully acknowledges the funding from UNITEN R\&D Sdn. Bhd. and Tenaga Nasional Berhad under TNB Seed Fund U-TI-RD-18-07.

\section{REFERENCES}

[1] A. Irawan, et al., "Label-Qos Switching Protocol For Quality of Service Assurance in Dynamic Swarm Robot Local Network," International Journal of Communication Systems, vol. 31, pp. 1-16, 2018.

[2] M. Arafah, et al., "Smart Systems Approach for Development of Explicit Congestion Marking and Traffic Engineering Model for Diffserv/MPLS Networks," Journal of Ambient Intelligence and Humanized Computing, pp. 1-11, 2018.

[3] M. Masood and M. M. Fouad, "Analysis of Artificial Intelligence-Based Metaheuristic Algorithm for MPLS Network Optimization," in 2018 20th International Conference on Transparent Optical Networks (ICTON), 2018, pp. 1-4.

[4] M. Imran, et al., "Design and Simulation of Traffic Engineering Using MPLS in GNS3 Environment," in 2018 Second International Conference on Computing Methodologies and Communication (ICCMC), 2018, pp. 1026-1030.

[5] E. N. Lallas, "A Survey On All Optical Label Swapping Techniques: Comparison And Trends," Optical Switching and Networking, vol. 31, pp. 22-38, 2019.

[6] S. Patil and M. S. Subhedar, "Optimizing MPLS Tunnel Creation Performance by Using SDN," Soft Computing and Signal Processing, pp. 527-536, 2019. 
[7] S. Patil and M. S. Subhedar, "Analysing MPLS Performance by SDN," Emerging Technologies in Data Mining and Information Security, pp. 589-598, 2019.

[8] M. M. Pijanka and G. W. Różański, "Mobile MPLS-TP - Support The Mobility of Terminal Devices Using OAM Channel," International Journal of Electronic and Telecommunications, vol. 62, pp. 215-223, 2016.

[9] R. Q. Shawl, et al., "A Review: Multi Protocol Label Switching (MPLS)," Int. Journal of Engineering Research and Applications, pp. 2248-9622, 2014.

[10] P. Biradar and T. Purushottama, "Design of Traffic Engineered Multi-Protocol Label Switching-Transport Profile (MPLS-TP) for The Enhancement of Quality of Service." International Journal of Research - GRANTHAALAYAH, vol. 5, pp. 58-67, 2017.

[11] M. Tabassum, et al., "Implementation and Performance Evaluation of Advance Metering Infrastructure for BorneoWide Power Grid," Frontiers in Energy, pp. 1-20, 2016.

[12] A. Sydney, et al., "Using GENI for Experimental Evaluation of Software Defined Networking in Smart Grids," Computer Networks, vol. 63, pp. 5-16, 2014.

[13] F. Kamoun and F. Outay, "IP/MPLS Networks with Hardened Pipes: Service Concepts, Traffic Engineering and Design Considerations," Journal of Ambient Intelligence and Humanized Computing, pp. 1-8, 2018.

[14] S. M. Blair, et al., "Modeling and Analysis of Asymmetrical Latency in Packet-Based Networks for Current Differential Protection Application," IEEE Transactions on Power Delivery, vol. 33, pp. 1185-1193, 2018.

[15] T. Rahman, et al., "Teleprotection with MPLS Ethernet Communications-Development and Testing of Practical Installations," in 2018 71st Annual Conference for Protective Relay Engineers (CPRE), 2018, pp. 1-18.

[16] I. Aldaya, et al., "Optical Switch Emulation in Programmable Software Router Testbed," Photonic Network Communications, vol. 25, pp. 10-23, 2013.

[17] Z.-k. Wang, et al. "Load-Balancing High-Performance Router Testbed Based On Openflow And Click," in 2010 International Conference on Computational Intelligence and Software Engineering (CiSE), 2010, pp. 1-4.

[18] L. Xu, et al., "MINOS: Regulating Router Dataplane Actions in Dynamic Runtime Environments," in Proceedings of the ACM Turing 50th Celebration Conference-China, 2017, pp. 1-10.

[19] M. M. Tajiki, et al., "SDN-Based Resource Allocation in MPLS Networks: A Hybrid Approach," Concurrency Computation, pp. 1-13, 2018.

[20] P. Goswami, et al., "On Methodologies to Estimate Optical-Layer Power Consumption and Cost for Long-Haul WDM Networks with Optical Reach Constraint," Photonic Network Communications, vol. 29, pp. 12-31, 2015.

[21] M. A. Kourtis, et al., "Enhancing VNF Performance by Exploiting SR-IOV and DPDK Packet Processing Acceleration," in 2015 IEEE Conference on Network Function Virtualization and Software Defined Network, NFVSDN 2015, 2016, pp. 74-78.

[22] A. Sgambelluri, et al., "Experimental Demonstration of Segment Routing," Journal of Lightwave Technology, vol. 34, pp. 205-212, 2016.

[23] S. M. Blair, et al., "Demonstration and Analysis of IP/MPLS Communications for Delivering Power System Protection Solutions Using IEEE C37. 94, IEC 61850 Sampled Values, and IEC 61850 GOOSE Protocols," in CIGRE Session $45-45^{\text {th }}$ International Conference on Large High Voltage Electric Systems 2014, August 2014, pp. 1-8, 2014.

[24] D. Posch, et al., "Emulating NDN-based Multimedia Delivery," in The 7th International Conference on Multimedia Systems, 2016, pp. 270-273.

[25] V. Sivaraman, et al., "Greening Router Line-Cards via Dynamic Management of Packet Memory," IEEE Journal on Selected Areas in Communications, vol. 34, pp. 3843-3853, 2016.

[26] V. Gupta, et al., "Developing Small Size Low-Cost Software-Defined Networking Switch Using Raspberry Pi," in $50^{\text {th }}$ Annual Convention of Computer Society of India: Next-Generation Networks, 2018, pp. 147-152.

[27] P. Lech and P. Włodarski, "IoT WiFi Home Network Stress Test," in $8^{\text {th }}$ International Conference on Image Processing and Communications Challenges, 2017, pp. 247-254.

[28] A. Zabri, et al., "Video Monitoring Application Using Wireless Sensor Node with Various External Antenna," Indonesian Journal of Electrical Engineering and Computer Science, vol. 6, pp. 148-154, 2017.

[29] K. Kalaithasan, et al., "Internet of Things Application in Monitoring Sick Building Syndrome," Indonesian Journal of Electrical Engineering and Computer Science, vol. 12, pp. 505-512, 2018.

[30] B. Rainer, et al., "A Low-Cost NDN Testbed on Banana Pi Routers," IEEE Communications Magazine, vol. 54, pp. 105-111, 2016.

\section{BIOGRAPHIES OF AUTHORS}

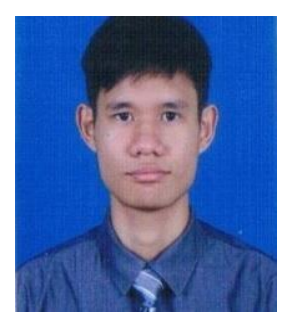

Muhammad Haqeem bin Mohd Nasir received the Bachelor of Electrical and Electronic Engineering degree from Universiti Tenaga Nasional (UNITEN) in 2018. He is currently doing his Master of Electrical Engineering in UNITEN while working as Research Engineer there. 


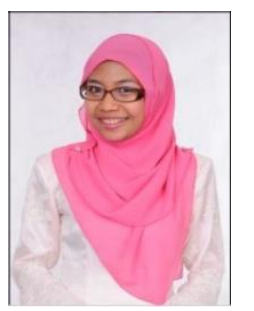

Nurul Asyikin Mohd. Radzi received her PhD. in Engineering, MEE and BEEE (Hons.) from Universiti Tenaga Nasional in the year 2013, 2010 and 2008, respectively. She is currently working as a senior lecturer in Department of Electronics and Communication Engineering, Universiti Tenaga Nasional. Her research interests include, optical communication and quality of service. She has contributed 50 technical papers in various journals and conferences. She is a senior member of IEEE Photonics Society and Chartered Engineer for IET.

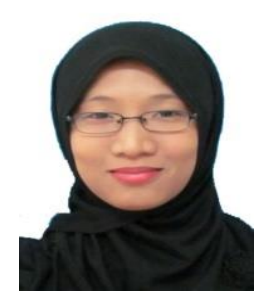

Wan Siti Halimatul Munirah Wan Ahmad received all her three degrees (the B.Eng. degree in Electronic Engineering majoring in Multimedia, M. Eng. Sc. and Ph. D.) from Multimedia University (MMU), Malaysia. She is currently a postdoctoral researcher at Universiti Tenaga Nasional (UNITEN), under UNITEN R\&D Sdn Bhd (URND), in modelling an algorithm for traffic scheduling project. Her main research interests are in the area of medical image analysis, content-based image retrieval, segmentation, feature extraction and data mining.

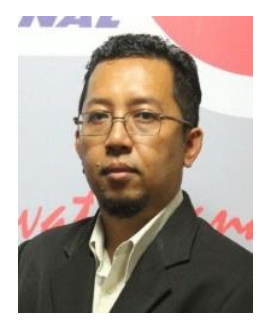

Fairuz Abdullah received the B.Eng. degree in electronics from Universiti Tenaga Nasional, Malaysia, in 2001. He then completed M.Sc. in network and communication engineering from Universiti Putra Malaysia in 2004 and his doctoral degree in fibre laser sensor from Universiti Tenaga Nasional in 2012. He is currently an associate professor in Department of Electronics \& Communication Engineering, College of Engineering, Universiti Tenaga Nasional. His research interest is in optical communications, fibre lasers, optical amplifiers and fibre optic sensors. To date, he has published more than 80 journal and conference papers. He has also been an active member of IEEE Photonic Society.

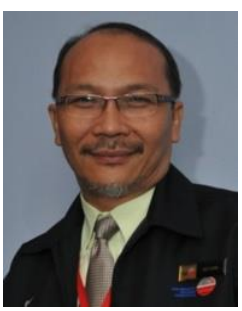

Prof. Ir. Dr. Md. Zaini Bin Jamaludin received his Diploma in Electrical and Electronic Engineering from Institute Technology Mara (know University Technology Mara, UiTM) in 1983, the B.Sc. Degree in Electrical Engineering from University of Miami, Florida, USA, in 1986, and M.Sc. in Electronic (Medical System) from University of Hertfordshire, UK, in 1994 and Ph.D. in Network Communication Engineering from Universiti Putra Malaysia 2007. He worked in Motorola Malaysia Sdn. Bhd in 1984 and 1986 as Assistant Engineer and Product Engineer respectively. He then joined UiTM in 1990, as Lecturer in the Faculty of Electrical Engineering. In 1997 he joined GITN Sdn. Bhd, the Malaysian e-government network service provider as a senior executive in the network security department. Joint Digicert Sdn. Bhd to set-up the first Certification Authority, company that issue the Digital Certificate for secured online transaction and appointed the Chief Operating Officer in 2000. He then joined the Department of Electronics and Communication Engineering, College of Engineering, Universiti Tenaga Nasional in 2001 as a Senior Lecturer. He was main person responsible in setting up the Spin off company under UNITEN that is UNITEN R\&D Sdn Bhd (URND) and was appointed as Managing Director of URND from 2013 until 2017. He is currently a Professor at Department of Electronics and Communication Engineering and now seconded to Institute of Power Engineering (IPE). He work and interest include, Photonics devices and Sensors, Optical network, Secured remote data acquisition systems, RF radiation (GSM, Mobile base station), and Ethernet Passive Optical Networks. He is an active researcher with more than RM7.0 million worth of research grants secured from various Research funding and agencies such as LRGS, eScience Fund, IRPA, PRGS, TNBR, MCMC and JICA. He has authored and coauthored more than 100 research papers in journals and conference proceedings. Prof. Md Zaini Jamaludin has been an active executive committee member for of IEEE Photonics Society, International Conference on Photonics (ICP) since 2004 as Conference Chairmen as well as committee members, including as Chairman in 2007-2008, and member of IEEE Malaysia for the past 15 years, recently promoted to Senior Member of IEEE and Member of IET since 2010. 